\title{
Inverted Rearfoot posture in subjects with coexisting patellofemoral osteoarthritis in medial knee osteoarthritis: an exploratory study
}

\author{
Hirotaka lijima ${ }^{1,2,3^{*}}$, Hiroshi Ohi ${ }^{4,5}$, Naoto Fukutani ${ }^{2}$, Tomoki Aoyama ${ }^{2}$, Eishi Kaneda ${ }^{6}$, Kaoru Abe ${ }^{4}$,
} Masaki Takahashi ${ }^{1}$ and Shuichi Matsuda ${ }^{7}$

\begin{abstract}
Background: While abnormal rearfoot posture and its relationship to patellofemoral (PF) pain has been thoroughly discussed in the literature, its relationship to patellofemoral osteoarthritis (PFOA) has not been determined. This study aimed to examine whether rearfoot posture is associated with a higher prevalence of radiographic PFOA in a compartment-specific manner in patients with medial tibiofemoral osteoarthritis (TFOA).

Methods: Participants from orthopedic clinics ( $n=68$, age 56-90 years, 75.0\% female), diagnosed with radiographic medial TFOA (Kellgren/Lawrence $[K / L$ ] grade $\geq 2$ ) were included in this study. The presence of PFOA and static rearfoot posture were evaluated using a radiographic skyline view and a footprint automatic measurement apparatus, respectively. The relationship between rearfoot posture and PFOA was examined using analysis of covariance and propensity scoreadjusted logistic regression analysis.

Results: On average, patients with coexisting PFOA and medial TFOA $(n=39)$ had an inverted calcaneus $3.1^{\circ}$ greater than those with isolated medial TFOA $(n=29)$. Increased calcaneus inverted angle was significantly associated with a higher probability of the presence of medial PFOA (odds ratio: 1.180, 95\% confidence interval: [1.005, 1.439]; $p=0.043$ ). Calcaneus inverted angle was not associated with higher odds of lateral PFOA presence based on the adjusted values.

Conclusions: The presence of an inverted rearfoot was associated with PFOA. Although these findings do not clearly indicate a biomechanical link between rearfoot posture and PFOA, this study shed light on the potential relationship between altered rearfoot posture and PFOA, as can be seen between rearfoot abnormality and PF pain.
\end{abstract}

Keywords: Rearfoot, Patellofemoral osteoarthritis, Inversion

\section{Background}

Knee osteoarthritis $(\mathrm{OA})$ is the leading cause of knee pain and disability worldwide [1]. Patellofemoral (PF) osteoarthritis (PFOA) is an under-recognized, yet important, subgroup of knee OA [2,3]. Depending on the source population and definition of OA, PFOA is present in $32-57 \%$ of adults [4] and commonly occurs in

\footnotetext{
* Correspondence: iijima.hirotaka.4m@yt.sd.keio.ac.jp

'Department of System Design Engineering, Keio University, Yokohama, Japan

${ }^{2}$ Department of Physical Therapy Human Health Sciences, Graduate School of Medicine, Kyoto University, Kyoto, Japan

Full list of author information is available at the end of the article
}

combination with tibiofemoral (TF) OA (TFOA) [5-7]. The risk factors in OA pathogenesis vary according to the affected compartment [8-10] and targeted interventions for PF joint disease are required owing to the unique biomechanics of the PF joint $[2,11]$. However, the factors that contribute to its development and the effective management of this common and potentially debilitating condition [3] have not been elucidated.

The rearfoot affects the biomechanical alignment of the lower limb [12,13], and therefore, it has the potential to lead to proximal diseases including PFOA [14]. Tibeiro et al. hypothesized that excessive eversion of the rearfoot can lead to increased tibial and femoral internal 
rotation, subsequently resulting in higher lateral PF cartilage stress due to increased knee valgus and quadriceps angle [15], although this has not proven yet. The relationship between altered foot posture during gait and PF pain was thoroughly discussed in the literature [16] and rearfoot abnormalities may be associated with PF pain [17]. The clinical symptoms and functional limitations between PFOA and PF pain in adolescents and young adults $[2,18]$ are similar, and approximately $70 \%$ of cases have radiographic PFOA in subjects (aged $>40$ years) with PF pain [5]. Therefore, it is possible that an altered rearfoot posture would be modifiable factors associated with PFOA, as can be seen between rearfoot posture and PF pain. Although foot orthoses were effective in improving anterior knee pain in patients with isolated lateral PFOA [19], we are not aware of any studies that investigated the relationship between rearfoot posture and PFOA.

Static rearfoot posture can be easily evaluated in the clinical setting without sophisticated equipment and has been used in assessments of patients with PF pain [20]. Investigating rearfoot posture in patients with coexisting PFOA may assist clinicians to better understand the effect of rearfoot posture on PF pathology and may add to the limited evidence of studies with PFOA population. Thus, this study aimed to examine whether altered static rearfoot posture is associated with higher prevalence of radiographic PFOA in patients with medial TFOA in a compartment-specific manner. Such a relationship would indicate the existence of a biomechanical association, necessitating a prospective cohort study to find modifiable risk factors for the incidence and progression of PFOA.

\section{Methods}

\section{Participants}

Participants of this exploratory study were recruited from the 12-month follow-up period of a prospective cohort of subjects described in a previous study, which investigated the clinical impact of coexisting PFOA in patients with medial TFOA [21]. Briefly, 143 patients with medial knee OA were recruited from a community orthopedic clinic in February 2014, and were followed up for 12 months. The patients, diagnosed by their attending physician, were recruited through advertisements and followed up for 12 months. The inclusion criteria were (i) age $\geq 50$ years; (ii) radiographic OA (i.e. Kellgren/Lawrence [K/L] [22] grade $\geq 2$ ) primarily in the medial TF compartment in one or both knees, as evaluated by weight-bearing anteroposterior radiographs; and (iii) the ability to walk independently on a flat surface without any ambulatory assistive device. Subjects at baseline were included if they had medial TFOA, regardless of PFOA status. No restriction was imposed on laterality; both patients with bilateral and unilateral radiographic knee OA were included in this study. The exclusion criteria were: (i) a history of knee surgery, (ii) inflammatory arthritis, (iii) periarticular fracture, (iv) current neurological problems, or (v) lateral TFOA. Lateral TFOA was defined as a knee having a $\mathrm{K} / \mathrm{L}$ grade $\geq 1$ along with joint space narrowing $(\mathrm{JSN})>0$ in the lateral compartment with JSN $=0$ in the medial compartment [23]. In other words, only patients who had a more severe radiographic disease in the medial compartment compared to the lateral compartment (i.e., isolated medial TFOA or mixed medial and lateral TFOA) were included in this study. Since medial and lateral knee OA have distinct characteristics [24], and most knee OA is the medial type in Japan $[25,26]$, lateral TFOA (i.e., lateral OA severity > medial OA severity) was excluded in this study. The Ethical Committee of Kyoto University approved this study (approval number: E1923). Written informed consent was obtained from all participants at baseline and at 12 months follow-up.

\section{Radiographic PF joint disease severity}

The radiographic data of the lateral and skyline views at baseline were obtained from all participants. If clinical symptoms worsened within the 12-month follow-up period, participants underwent repeat radiography. Detailed methods of radiographic evaluation of disease severity in the PF joints were described elsewhere [21]. Briefly, a single trained examiner $(\mathrm{HI})$ assessed radiographic severity for the $\mathrm{PF}$ joint using the $\mathrm{K} / \mathrm{L}$ grading system adapted to the lateral and medial facets of the PF joint. Presence of PFOA was defined as knee with K/L grade 2 in skyline view or osteophytes 1 in lateral view. We have previously reported excellent intra-rater reliability for such radiographic evaluation (Kappa: 0.80) [21].

\section{Static Rearfoot posture}

Static foot posture was evaluated in January 2015 using a three-dimensional automatic footprint measurement apparatus (CUTE, JMS-2100CU; Dream GP Inc., Osaka, Japan) [27, 28]. This foot scanning system is based on laser line triangulation, where the measuring head moves around a single foot in an oval-shaped trajectory [29]. The laser scanner rotates around the patient's foot and measures more than 30,000 points, including the ankle, instep, heel, toes, as well as the sole, thereby precisely re-creating the patient's foot shape. This scanning system has a high accuracy for measuring foot posture. The measurement errors of foot length and foot width are $0.27-0.36 \mathrm{~mm}$ (accuracy $\pm 0.2 \%$ ) and $0.51-1.22 \mathrm{~mm}$ (accuracy $\pm 0.5 \%$ ), respectively [29].

Prior to each capture session, the patient was asked to stand on bare feet with shoulder-width apart. This allowed $50 \%$ of their body weight to be placed on each 
foot during assessment. Round, black seal markers, which corresponded to 2 anatomical landmarks (i.e., bottom of the calcaneal tuberosity and enthesis of the Achilles tendon) to detect foot alignment, were attached to the skin (Fig. 1). After the measurements, foot length and calcaneus inverted angle relative to the floor were automatically calculated by the system according to the attached round black seal markers. As a clinical frame of reference, calcaneus inverted angle relative to the floor were categorized based on value of foot posture index subcategory "inversion/eversion of the calcaneus" [30] as follows: everted calcaneus (calcaneus angle $\leq-5^{\circ}$ ); normal calcaneus $\left(-5^{\circ}<\right.$ and $\left.\leq 5^{\circ}\right)$; and inverted calcaneus (calcaneus angle $>5^{\circ}$ ). Throughout the manuscript, "inversion/eversion" indicates posture on a single frontal plane, which is a part of triplane motion "supination/ pronation".

\section{Covariates}

Data on age, sex, and height were self-reported by patients. Patients wearing clothes without shoes were weighed on a scale. Body mass index (BMI) was calculated by dividing the weight by height squared. Radiographic medial TFOA severity and anatomical axis angle (AAA) with sex-specific correction [31] were assessed in the anteroposterior short view in the weight-bearing position. The intra-rater reliability was excellent for evaluating the TFOA K/L grade (Kappa: 0.80) and measuring the AAA (intra-class correlation coefficient [ICC]: $0.98)$. Varus thrust was evaluated according to previously described methods [32, 33]. Two physical therapists judged the presence of lateral movement of the tibial tuberosity relative to hip and ankle. This resulted in an increase in varus alignment during initial contact with the mid-stance of the stance phase with self-selected speed. We have previously reported good interrater reliability (Kappa: 0.73) for evaluating varus thrust assessment [32, 33]. A trained physical therapist passively measured the flexion and extension range of motion (ROM) of the affected knee joint through standard goniometric procedures according to previously validated methods [34].

\section{Statistical analyses}

To minimize any bias produced by similarities between the knees of the same patient, only one knee per patient was analyzed, which was designated as the "index knee." The index knee was defined as the more painful knee in the present or past. Descriptive statistics were calculated as mean and standard deviation (SD) for continuous variables and as proportion for dichotomous/categorical variables. We performed univariate analysis using Student's $t$-test for parametric continuous variables and Fisher's exact test for dichotomous/categorical variables and compared the differences in rearfoot posture between knees with and without PFOA. Subsequently, the values of calcaneus inverted angle were compared using analysis of covariance. Covariates included age, sex, BMI, TF joint $\mathrm{K} / \mathrm{L}$ grade, corrected AAA, presence of varus thrust, and knee flexion ROM. These covariates were chosen based on clinical judgment and previous studies investigating factors associated with PFOA or rearfoot posture [21,35-37]. The normality of calcaneus inverted angle was assessed using the Shapiro-Wilk test and the homogeneity of the variances between patients with and without PFOA was confirmed using the F-test.

We further performed multiple logistic regression analyses and calculated odds ratios (ORs) and their 95\% CIs. Logistic regression analyses were performed first with an unadjusted model and then with a propensity adjusted model. Due to the small sample size, we used propensity score adjustment including the above covariates. Data analyses were performed with JMP Pro 12.2 (SAS Institute, Cary, NC, USA). P-values $<0.05$ were considered statistically significant.
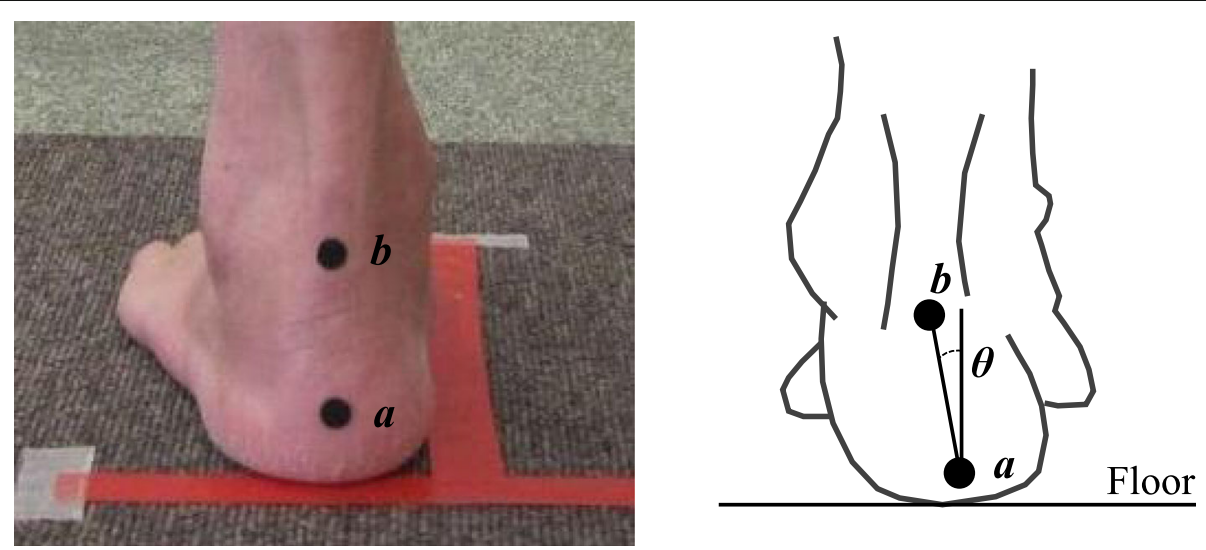

Fig. 1 Measurement of calcaneus inverted angle relative to the floor $(\theta)$. Calcaneus inverted angle relative to the floor was automatically calculated by the system according to the attached round black seal markers ( $a$ bottom of the calcaneal tuberosity; $b$ enthesis of the Achilles tendon) 


\section{Results}

Figure 2 shows flowchart describing the inclusion of study participants. One-hundred-forty-three patients with medial knee OA were enrolled at baseline period (February 2014). Of these patients, 75 were excluded from this study; 41 could not be contacted or declined follow up for non-specific reasons; and 34 were excluded because of missing data on patient's characteristics, radiography, and rearfoot posture at the 12 months follow up period (January 2015). Thus, our final sample included 68 patients $(47.6 \%$ of the initial cohort) at 12 months follow-up period. Baseline characteristics were compared between included and excluded patients, and no significant differences were found between the two groups in terms of demographic characteristics and radiographic disease severity at baseline period (data not shown). Of the 68 patients who completed the study (age 56-90 years; $75.0 \%$ female), 48 (70.6\%) had mild diseases with $\mathrm{K} / \mathrm{L}$ grade $=2$ in their index knee (Table 1 ). Thirty-eight (55.9\%) of these patients had PFOA.

Table 2 shows the comparison of calcaneus inverted angle and calcaneus alignment in knees with and without PFOA. Calcaneus inverted angle in patients with coexisting PFOA was higher than those with isolated TFOA (1.046 \pm 5.053 vs. $-2.245 \pm 5.648 ; \quad p=0.014)$. Patients with coexisting PFOA on average had an inverted calcaneus $3.1^{\circ}$ greater than those with isolated medial TFOA after adjusting for age, sex, BMI, TF joint
$\mathrm{K} / \mathrm{L}$ grade, corrected AAA, presence of varus thrust, and knee flexion ROM $(p=0.047)$. Patients with coexisting PFOA had a higher prevalence of inverted calcaneus $(15.4 \%$ vs. $6.9 \%)$ and lower prevalence of everted calcaneus (12.8\% vs. $27.6 \%)$, although these calcaneus alignments did not significantly differ between the two groups $(p=0.218)$.

Logistic regression analyses (Table 3 ) revealed that rearfoot posture was associated with PFOA in a non-compartment specific manner. Calcaneus inverted angle was significantly associated with higher odds of the presence of any $(\mathrm{OR}=1.134,95 \%$ CI [1.013, 1.291], $p=0$. 028) and medial PFOA (OR $=1.180,95 \%$ CI $[1.005,1$. 439], $p=0.043)$; however, significant relationships were not confirmed mixed $(\mathrm{OR}=1.135,95 \% \mathrm{CI}[0.958,1.406]$; $p=0.147)$ and lateral PFOA $(\mathrm{OR}=1.078,95 \% \mathrm{CI}[0.965$, 1.213], $p=0.183)$.

\section{Discussion}

This exploratory study showed that patients with coexisting PFOA and medial TFOA on average had an inverted calcaneus $3.1^{\circ}$ greater than those with isolated medial TFOA after adjusting for covariates, although approximately $70 \%$ of patients in both groups had a normal range of calcaneus angle. Increased calcaneus inverted angle was significantly associated with higher odds of any and medial PFOA and likely to be associated with higher odds of the presence of mixed and lateral

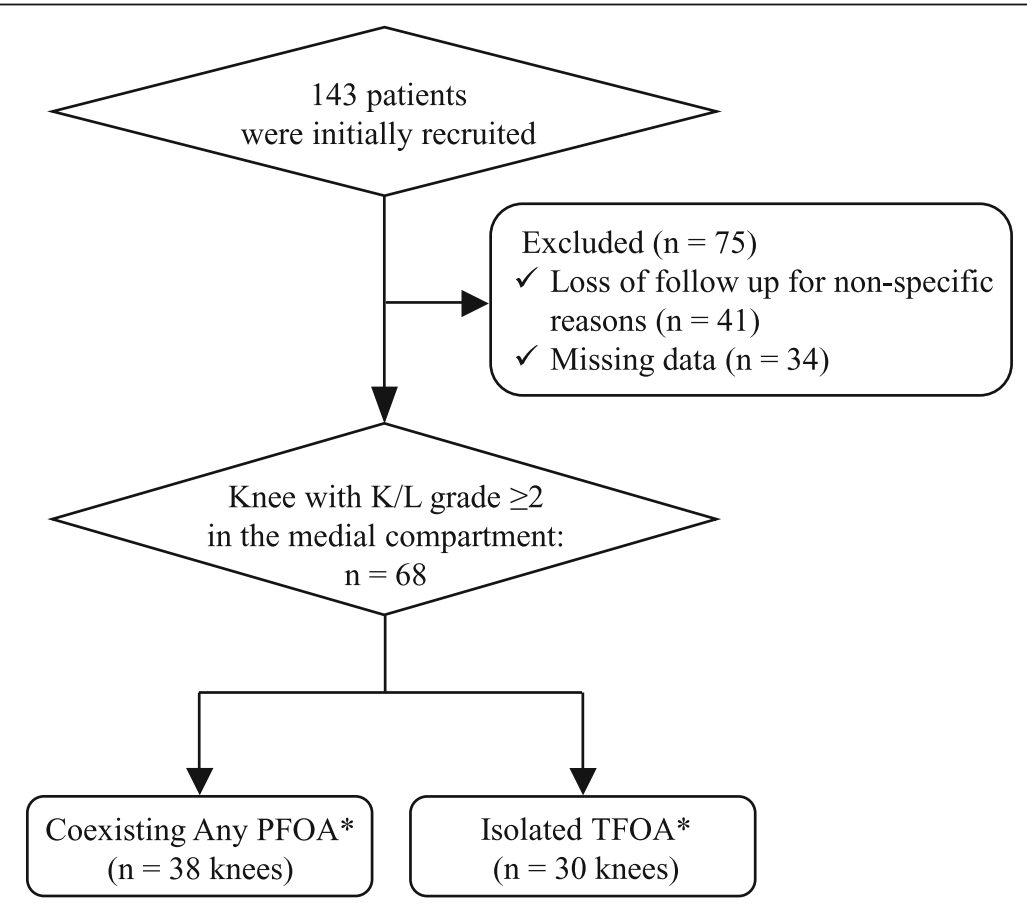

Fig. 2 Flowchart showing the inclusion of participants in the study. *Presence of PFOA was defined as knee with $K / L$ grade 2 in skyline view or osteophytes 1 in lateral view [21] based on baseline radiography. If clinical symptoms worsened within the 12-month follow-up, participants underwent repeat radiography that is used for evaluation of the PFOA presence 
Table 1 Patients' characteristics at follow up period $(n=68)^{a}$

\begin{tabular}{ll}
\hline Age, years & $74.69 \pm 7.785$ \\
Female, no. (\%) & $51(75.0)$ \\
Body mass index, kg/m² & $24.14 \pm 3.753$ \\
Corrected anatomical axis angle, degrees & $176.2 \pm 4.951$ \\
$\quad$ Presence of varus alignment, no. (\%) ${ }^{\mathrm{a}}$ & $55(80.9)$ \\
Medial tibiofemoral joint K/L grade, no. (\%) & \\
$\quad$ Grade 2 & $48(70.6)$ \\
$\quad$ Grade 3 & $11(16.2)$ \\
$\quad$ Grade 4 & $9(13.2)$ \\
Presence of varus thrust, no. (\%) & $12(17.6)$ \\
Knee range of motion, degrees & \\
$\quad$ Extension ${ }^{\mathrm{c}}$ & $-6.471 \pm 6.482$ \\
$\quad$ Flexion & $140.8 \pm 12.17$ \\
Presence of any PFOA, no. (\%) & $38(55.9)$ \\
Presence of mixed PFOA, no. (\%) & $19(27.9)$ \\
Presence of medial PFOA, no. (\%) & $22(68.8)$ \\
Presence of lateral PFOA, no. (\%) & $26(32.4)$ \\
Coexisting medial TFOA and any PFOA, no. (\%) & $38(55.9)$ \\
Calcaneus inverted angle, degrees ${ }^{\mathrm{d}}$ & $-0.357 \pm 5.522$ \\
\hline
\end{tabular}

K/L grade: Kellgren/Lawrence grade; PFOA: patellofemoral osteoarthritis; TFOA: tibiofemoral osteoarthritis

Except where otherwise indicated, values are mean \pm SD

a Varus alignment is defined as corrected anatomical axis angle $<179$ degrees

blf participants did not get worse their clinical symptoms within the 12-month

follow-up period, radiography at baseline was used for K/L grade assessment

${ }^{\mathrm{C}} \mathrm{A}$ negative value indicates that the knee is flexed

${ }^{d} A$ positive value indicates inversion direction of the calcaneus

PFOA. The association between rearfoot alignment was in the same direction for medial or lateral PFOA, thereby rearfoot alignment appears not to be associated with compartmental distribution of PFOA. Potential risk factors associated with PFOA involve patellar alignment relative to trochlea; muscle weakness, such as in the quadriceps; and abnormal biomechanics [38]. While an extensive literature review found similarities in clinical symptoms, structure, and physical function between patients with PF pain and PFOA, none of the included studies examined rearfoot posture in patients with PFOA [18]. Thus, this study is the first to show that rearfoot posture may be a potential modifiable factor associated with PF joint disease.

Excessive rearfoot eversion is suggested to lead to tibial and femoral internal rotation, subsequently resulting in increased quadriceps angle and higher lateral PF cartilage stress [15] in accordance with "law of valgus" (i.e. varus alignment increases the medial $\mathrm{PF}$ force and valgus alignment increases the lateral PF force) [39]. If this biomechanical theory is correct, everted and inverted calcaneus are associated with lateral and medial PFOA, respectively. This theory is supported by the significant relationship between calcaneus inverted angle and the presence of medial PFOA shown by the findings. However, calcaneus inverted angle is likely associated with lateral PFOA given that the lower limit of $95 \% \mathrm{CI}$ of OR is near 1.0. These findings do not clearly support a biomechanical link between inverted rearfoot alignment and medial compartment-specific PFOA. Thus, inverted calcaneus may represent a clinical feature of multicompartmental disease. Further studies examining the biomechanical link between rearfoot abnormality and PFOA are warranted to support the findings from this exploratory study.

We found that calcaneus inverted angle was associated with a higher prevalence of medial PFOA. Conflicting evidence linking foot eversion and PF exists, although excessive foot eversion and its relationship to PF pain have been discussed in literature. For example, Powers et al. found that patients with PF pain exhibited increased rearfoot inversion compared to those without PF pain in younger adults when examined using a goniometer [20]. The presence of both PF pain and altered foot posture can lead to this progression given that PF pain in younger adults is suggested to be a precursor to

Table 2 Comparison of calcaneus inverted angle and calcaneus alignment in knees with and without PFOA $(n=68)$

\begin{tabular}{|c|c|c|c|c|c|}
\hline Variables & $\begin{array}{l}\text { Coexisting Any PFOA } \\
(n=38 \text { knees) }\end{array}$ & $\begin{array}{l}\text { Isolated TFOA } \\
(n=30 \text { knees })\end{array}$ & $p$-value ${ }^{\dagger}$ & Difference in mean $(95 \% \mathrm{Cl})^{\dagger+}$ & $p$-value \\
\hline Calcaneus inverted angle, degrees§ & $1.046 \pm 5.053$ & $-2.245 \pm 5.648$ & 0.014 & $3.109(0.037,6.181)$ & 0.047 \\
\hline Calcaneus alignment, no (\%)§§ & & & 0.218 & & \\
\hline Everted calcaneus & $5(13.2)$ & $8(26.7)$ & & & \\
\hline Normal calcaneus & $27(71.1)$ & $20(66.7)$ & & & \\
\hline Inverted calcaneus & $6(15.8)$ & $2(6.7)$ & & & \\
\hline
\end{tabular}

PFOA: patellofemoral osteoarthritis; TFOA: tibiofemoral osteoarthritis; $95 \% \mathrm{Cl}$ : 95\% confidence interval

Except where otherwise indicated, values are mean \pm SD

${ }^{\dagger}$ Based on Student $t$-test (calcaneus inverted angle) and the Fisher's exact tests (calcaneus type) between two groups

${ }^{+\dagger}$ Adjusted for age, (continuous), sex (0: male, 1: female), body mass index (continuous), tibiofemoral joint Kellgren/Lawrence grade (continuous), corrected

anatomical axis angle (continuous), presence of varus thrust (0: absence, 1: presence), and knee flexion range of motion (continuous)

${ }^{\S} \mathrm{A}$ positive value indicates inversion direction of the calcaneus.

$\S \S$ Inverted calcaneus: calcaneus angle $\leq-5$ degree; normal calcaneus: -5 degree $<$ and $\leq 5$ degree; everted calcaneus: calcaneus angle $>5$ degree.

Bold type represents a statistically significant result 
Table 3 Results of binary logistic regression analysis of the association between calcaneus inverted angle and the presence of PFOA $(n=68)$

\begin{tabular}{|c|c|c|c|}
\hline \multirow[t]{2}{*}{ Independent variable } & \multirow[t]{2}{*}{ Dependent variable } & \multicolumn{2}{|l|}{ Odds ratio $(95 \% \mathrm{Cl})^{*}$} \\
\hline & & Crude model & Propensity adjusted model \\
\hline \multirow{4}{*}{$\begin{array}{l}\text { Calcaneus inverted angle, } \\
\text { per degrees }\end{array}$} & No PFOA $(n=30)$ vs. ANY PFOA $(n=38)$ & $1.118(1.018-1.245)^{\dagger}$ & $1.134(1.013-1.291)^{\dagger}$ \\
\hline & No PFOA $(n=30)$ vs. MIXED PFOA $(n=19)$ & $1.166(1.031-1.356)^{\dagger}$ & $1.135(0.958-1.406)$ \\
\hline & No PFOA $(n=30)$ vs. MEDIAL PFOA $(n=22)$ & $1.180(1.044-1.368)^{\dagger \dagger}$ & $1.180(1.005-1.439)^{\dagger}$ \\
\hline & No PFOA $(n=30)$ vs. LATERAL PFOA $(n=26)$ & $1.109(1.010-1.235)^{\dagger \dagger}$ & $1.078(0.965-1.213)$ \\
\hline
\end{tabular}

PFOA: patellofemoral osteoarthritis; $95 \% \mathrm{Cl}$ : 95\% confidence interval

${ }^{*}$ Adjusted for propensity to prescribe as a function of age, (continuous), sex (0: male, 1: female), body mass index (continuous), tibiofemoral joint Kellgren/ Lawrence grade (continuous), corrected anatomical axis angle (continuous), presence of varus thrust (0: absence, 1: presence), and knee flexion range of motion (continuous)

${ }^{\dagger} p<0.05 ;{ }^{++} p<0.01$

PFOA. However, due to the cross-sectional nature of this study, PFOA may develop first and altered rearfoot posture may be a consequence of PFOA. Bidirectional segmental relationship has been determined among foot, shank, thigh, and pelvis [40]; therefore, a prospective cohort study on the incidence of PFOA in patients with inverted rearfoot but without PFOA should be conducted. This is particularly important given that risk factors associated with the incidence and progression of PFOA have not been fully determined.

It should be noted that there was a large interindividual variability of calcaneus inverted angles, although patients with coexisting PFOA had an inverted calcaneus $3.1^{\circ}$ greater than those with isolated medial TFOA. Understanding these variabilities is important because interventions concerning foot orthoses targeting PF joint disease may lack clinical significance $[19,41]$ and evaluating individual rearfoot posture may facilitate pain reduction of foot orthoses. Sultive et al. found that increased inverted calcaneus during standing is a potential indicator of non-success in the treatment of foot orthoses for improving PF pain [42], indicating a substantial role for rearfoot posture on foot orthoses in targeting the PF joint.

The current study included patients with medial TFOA and compared rearfoot posture in patients with and without PFOA because mixed OA is common [5-7] and is likely to be more painful than those with isolated PFOA [43]. However, the observed relationship between varus thrust and the presence of PFOA in patients with medial knee OA may not be true for patients with isolated PFOA that was suggested as a precursor of mixed OA [44].

There are some limitations to be noted. First, the cross-sectional study design limits our ability to identify causality between inverted rearfoot posture and PFOA. Second, a foot scanning system was used for static measurements while standing. Evaluating dynamic rearfoot alignment through three-dimensional motion capture apparatus [45] may provide substantial information about the association between foot posture and PFOA with higher accuracy and reliability than static measure [46]. Furthermore, calcaneus inverted angle does not include the subtalar joint and may yield different values compared to traditional evaluation methods that use goniometers for evaluating rearfoot posture [47]. Nevertheless, this scanning system is advantageous because it has a high accuracy for measuring static foot posture [29] which can be clinically assessed in a short amount of time. Third, PFOA identification using radiographs is an important limitation. Radiographic assessment indirectly measures the cartilage and is less sensitive than MRI. This would lead to differences in the prevalence of coexisting PFOA. Specifically, patients with isolated TFOA may have cartilage damage in the PF joint without radiographic evidence of PFOA. Furthermore, radiographic views might be affected by knee position and the patellar alignment. Fourth, this study included subjects who did not undergo follow-up x-ray is an important limitation. Some of the individuals who did not have PFOA at baseline might have developed radiographic PFOA at followup without worsen of clinical symptoms, which might affect the relationship between rearfoot alignment and presence of PFOA. Finally, this study did not account for confounders of PFOA, such as the quadriceps muscle [48] and gait kinematics [48, 49]. These possible confounders need to be examined further using epidemiologic studies to elucidate the relationship between rearfoot posture and PFOA.

\section{Conclusions}

This exploratory study found that patients with coexisting PFOA on average had a $3.1^{\circ}$ more inverted calcaneus than those with isolated medial TFOA after adjustment for covariates. Increased calcaneus inverted angle was significantly associated with higher odds of the presence of any and medial PFOA, and likely to be associated with higher odds of the presence of mixed lateral PFOA. Further studies are warranted to elucidate the pathomechanics linking rearfoot and PF joint disease. 


\section{Abbreviations}

AAA: Anatomical axis angle; BMI: Body mass index; ICC: Intra-class correlation coefficient; K/L grade: Kellgren/Lawrence grade; OA: Osteoarthritis; OR: Odds ratio; PF: Patellofemoral; PFOA: Patellofemoral osteoarthritis; ROM: Range of motion; SD: Standard deviation; TFOA: Tibiofemoral osteoarthritis

\section{Acknowledgments}

The authors thank Ms. Yuko Yamamoto, Mr. Masakazu Hiraoka, Mr. Kazuyuki Miyanobu, and Mr. Masashi Jinnouchi (Nozomi Orthopaedic Clinic, Hiroshima) for assistance and advice.

\section{Funding}

This study was supported by a Grant-in-Aid for Scientific Research (grant no. $16 \mathrm{dk0110007h0003)}$ from the Japan Society for the Promotion of Science (https://www.jsps.go.jp/).

\section{Availability of data and materials}

The datasets used and analyzed during the current study available from the corresponding author on reasonable request.

\section{Authors' contributions}

All authors have made substantial contributions to (1) substantial contributions to research design, or the acquisition, analysis or interpretation of data; (2) drafting the paper or revising it critically; (3) approval of the submitted and final versions; and (4) agreed to be accountable for all aspects of the work. The specific contributions of the authors are as follows: (1) Conception and design of the study: $\mathrm{HI}, \mathrm{HO}, \mathrm{NF}, \mathrm{TA}$, EK, and SM. (2) Analysis and interpretation of the data: HI, HO, NF, TA, EK, KA, MT, and SM. (3) Drafting of the article: HI, HO, NF, TA, EK, KA, MT, and SM. (4) Critical revision of the article for important intellectual content: HI, HO, TA. (5) Final approval of the article: HI, HO, NF, TA, EK, KA, MT, and SM. (6) Statistical expertise: HI, NF, and TA. (7) Obtaining of funding: TA and SM. (8) Collection and assembly of data: HI, HO, NF, TA, and EK.

\section{Ethics approval and consent to participate}

The ethical committee of Kyoto University approved the study (approval number: E1923), and written informed consent was obtained from all participants before their enrollment.

\section{Competing interests}

The authors declare that they have no competing interests.

\section{Publisher's Note}

Springer Nature remains neutral with regard to jurisdictional claims in published maps and institutional affiliations.

\section{Author details \\ 'Department of System Design Engineering, Keio University, Yokohama, Japan. ${ }^{2}$ Department of Physical Therapy Human Health Sciences, Graduate School of Medicine, Kyoto University, Kyoto, Japan. ${ }^{3} J a p a n$ Society for the Promotion of Science, Tokyo, Japan. ${ }^{4}$ Graduate School of Health and Welfare, Niigata University of Health and Welfare, Niigata, Japan. ${ }^{5}$ Ohi Manufacturing Co., Ltd., Kyoto, Japan. ${ }^{6}$ Nozomi Orthopedic Clinic, Hiroshima, Japan. ${ }^{7}$ Department of Orthopedic Surgery, Graduate School of Medicine, Kyoto University, Kyoto, Japan.}

Received: 8 January 2018 Accepted: 26 April 2018 Published online: 08 May 2018

\section{References}

1. Cross M, Smith E, Hoy D, Nolte S, Ackerman I, Fransen M, et al. The global burden of hip and knee osteoarthritis: estimates from the global burden of disease 2010 study. Ann Rheum Dis. 2014;73:1323-30.

2. Hinman RS, Crossley KM. Patellofemoral joint osteoarthritis: an important subgroup of knee osteoarthritis. Rheumatology (Oxford). 2007;46:1057-62.

3. Crossley KM, Hinman RS. The patellofemoral joint: the forgotten joint in knee osteoarthritis. Osteoarthr Cartil. 2011;19:765-7.

4. Hart HF, Stefanik JJ, Wyndow N, Machotka Z, Crossley KM. The prevalence of radiographic and MRI-defined patellofemoral osteoarthritis and structural pathology: a systematic review and meta-analysis. Br J Sports Med. 2017;51: 1195-208.
5. Hinman RS, Lentzos J, Vicenzino B, Crossley KM. Is patellofemoral osteoarthritis common in middle-aged people with chronic patellofemoral pain? Arthritis Care Res (Hoboken). 2014;66:1252-7.

6. McAlindon T, Zhang Y, Hannan M, Naimark A, Weissman B, Castelli W, et al. Are risk factors for patellofemoral and tibiofemoral knee osteoarthritis different? J Rheumatol. 1996:23:332-7.

7. Duncan RC, Hay EM, Saklatvala J, Croft PR. Prevalence of radiographic osteoarthritis-it all depends on your point of view. Rheumatology (Oxford). 2006:45:757-60

8. Cooper C, McAlindon T, Snow S, Vines K, Young P, Kirwan J, et al. Mechanical and constitutional risk factors for symptomatic knee osteoarthritis: differences between medial tibiofemoral and patellofemoral disease. J Rheumatol. 1994:21:307-13.

9. Cicuttini FM, Spector T, Baker J. Risk factors for osteoarthritis in the tibiofemoral and patellofemoral joints of the knee. J Rheumatol. 1997:24: 1164-7.

10. Schiphof D, van Middelkoop M, de Klerk BM, Oei EH, Hofman A, Koes BW, et al. Crepitus is a first indication of patellofemoral osteoarthritis (and not of tibiofemoral osteoarthritis). Osteoarthr Cartil. 2014;22:631-8.

11. Grelsamer RP, Klein JR. The biomechanics of the patellofemoral joint. J Orthop Sports Phys Ther. 1998;28:286-98.

12. Tiberio D. Pathomechanics of structural foot deformities. Phys Ther. 1988;68: 1840-9.

13. Resende RA, Deluzio KJ, Kirkwood RN, Hassan EA, Fonseca ST. Increased unilateral foot pronation affects lower limbs and pelvic biomechanics during walking. Gait Posture. 2015;41:395-401.

14. Powers CM. The influence of altered lower-extremity kinematics on patellofemoral joint dysfunction: a theoretical perspective. J Orthop Sports Phys Ther. 2003;33:639-46.

15. Tiberio D. The effect of excessive subtalar joint pronation on patellofemoral mechanics: a theoretical model. J Orthop Sports Phys Ther. 1987;9:160-5.

16. Barton CJ, Levinger $\mathrm{P}$, Menz HB, Webster KE. Kinematic gait characteristics associated with patellofemoral pain syndrome: a systematic review. Gait Posture. 2009:30:405-16

17. Aliberti S, Costa Mde S, Passaro Ade C, Arnone AC, Hirata R, Sacco IC. Influence of patellofemoral pain syndrome on plantar pressure in the foot rollover process during gait. Clinics (Sao Paulo). 2011;66:367-72.

18. Wyndow N, Collins N, Vicenzino B, Tucker K, Crossley K. Is There a biomechanical link between patellofemoral pain and osteoarthritis? A narrative review. Sports Med. 2016;46:1797-1808.

19. Collins NJ, Hinman RS, Menz HB, Crossley KM. Immediate effects of foot orthoses on pain during functional tasks in people with patellofemoral osteoarthritis: a cross-over, proof-of-concept study. Knee. 2017;24:76-81.

20. Powers CM, Maffucci R, Hampton S. Rearfoot posture in subjects with patellofemoral pain. J Orthop Sports Phys Ther. 1995;22:155-60.

21. lijima H, Fukutani N, Aoyama T, Fukumoto T, Uritani D, Kaneda E, et al. Clinical impact of coexisting patellofemoral osteoarthritis in Japanese patients with medial knee osteoarthritis. Arthritis Care Res (Hoboken). 2016;68:493-501.

22. Kellgren JH, Lawrence JS. Radiological assessment of osteo-arthrosis. Ann Rheum Dis. 1957;16:494-502

23. Altman RD, Gold GE. Atlas of individual radiographic features in osteoarthritis, revised. Osteoarthr Cartil. 2007;15(Suppl A):A1-56.

24. Butler RJ, Barrios JA, Royer T, Davis IS. Frontal-plane gait mechanics in people with medial knee osteoarthritis are different from those in people with lateral knee osteoarthritis. Phys Ther. 2011:91:1235-43.

25. Muraki S, Oka H, Akune T, Mabuchi A, En-yo Y, Yoshida M, et al. Prevalence of radiographic knee osteoarthritis and its association with knee pain in the elderly of Japanese population-based cohorts: the ROAD study. Osteoarthr Cartil. 2009;17:1137-43.

26. Muraki S, Akune T, En-Yo Y, Yoshida M, Suzuki T, Yoshida H, et al. Joint space narrowing, body mass index, and knee pain: the ROAD study (OAC1839R1). Osteoarthr Cartil. 2015:23:874-81.

27. Ohi H, lijima H, Aoyama T, Kaneda E, Ohi K, Abe K. Association of frontal plane knee alignment with foot posture in patients with medial knee osteoarthritis. BMC Musculoskelet Disord. 2017;18:246.

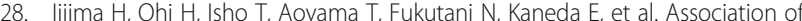
bilateral flat feet with knee pain and disability in patients with knee osteoarthritis: a cross-sectional study. J Orthop Res. 2017:35:2490-8.

29. Fujita H, Fukumoto S, Yoshida H, Wakasugi Y, Kano H. A 3D foot scanning system with a sensor head guided around the foot (Japanese). Transactions of the Institute of Systems, Control and Information Engineers. 2004;17:330-7. 
30. Redmond AC, Crosbie J, Ouvrier RA. Development and validation of a novel rating system for scoring standing foot posture: the foot posture index. Clin Biomech (Bristol, Avon). 2006;21:89-98.

31. Kraus VB, Vail TP, Worrell T, McDaniel G. A comparative assessment of alignment angle of the knee by radiographic and physical examination methods. Arthritis Rheum. 2005;52:1730-5.

32. Fukutani N, lijima H, Fukumoto T, Uritani D, Kaneda E, Ota K, et al. Association of Varus Thrust with Pain and Stiffness and activities of daily living in patients with medial knee osteoarthritis. Phys Ther. 2016;96:167-75.

33. lijima H, Fukutani N, Aoyama T, Fukumoto T, Uritani D, Kaneda E, et al. Clinical phenotype classifications based on static Varus alignment and Varus thrust in Japanese patients with medial knee osteoarthritis. Arthritis Rheumatol. 2015;67:2354-62.

34. Watkins MA, Riddle DL, Lamb RL, Personius WJ. Reliability of goniometric measurements and visual estimates of knee range of motion obtained in a clinical setting. Phys Ther. 1991;71:90-6.

35. Englund $M$, Lohmander LS. Patellofemoral osteoarthritis coexistent with tibiofemoral osteoarthritis in a meniscectomy population. Ann Rheum Dis. 2005;64:1721-6.

36. Cahue S, Dunlop D, Hayes K, Song J, Torres L, Sharma L. Varus-valgus alignment in the progression of patellofemoral osteoarthritis. Arthritis Rheum. 2004:50:2184-90.

37. lijima H, Fukutani N, Yamamoto Y, Hiraoka M, Miyanobu K, Jinnouchi M, et al. Association of varus thrust with prevalent patellofemoral osteoarthritis: a cross-sectional study. Gait Posture. 2017;58:394-400.

38. Crossley KM, Stefanik JJ, Selfe J, Collins NJ, Davis IS, Powers CM, et al. 2016 patellofemoral pain consensus statement from the 4th international patellofemoral pain research retreat, Manchester. Part 1: terminology, definitions, clinical examination, natural history, patellofemoral osteoarthritis and patient-reported outcome measures. Br J Sports Med. 2016;50:839-43.

39. Hungerford DS, Barry M. Biomechanics of the patellofemoral joint. Clin Orthop Relat Res. 1979:9-15.

40. Khamis S, Dar G, Peretz C, Yizhar Z. The relationship between foot and pelvic alignment while standing. J Hum Kinet. 2015;46:85-97.

41. Crossley KM, van Middelkoop M, Callaghan MJ, Collins NJ, Rathleff MS, Barton CJ. 2016 patellofemoral pain consensus statement from the 4th international patellofemoral pain research retreat, Manchester. Part 2: recommended physical interventions (exercise, taping, bracing, foot orthoses and combined interventions). Br J Sports Med. 2016:50:844-52.

42. Sutlive TG, Mitchell SD, Maxfield SN, McLean CL, Neumann JC, Swiecki CR, et al. Identification of individuals with patellofemoral pain whose symptoms improved after a combined program of foot orthosis use and modified activity: a preliminary investigation. Phys Ther. 2004;84:49-61.

43. Szebenyi B, Hollander AP, Dieppe P, Quilty B, Duddy J, Clarke S, et al. Associations between pain, function, and radiographic features in osteoarthritis of the knee. Arthritis Rheum. 2006;54:230-5.

44. Lankhorst NE, Damen J, Oei EH, Verhaar JAN, Kloppenburg M, BiermaZeinstra SMA, et al. Incidence, prevalence, natural course and prognosis of patellofemoral osteoarthritis: the cohort hip and cohort knee study. Osteoarthr Cartil. 2017:25:647-53.

45. Powers CM, Chen PY, Reischl SF, Perry J. Comparison of foot pronation and lower extremity rotation in persons with and without patellofemoral pain. Foot Ankle Int. 2002;23:634-40

46. Silva Dde O, Briani RV, Pazzinatto MF, Ferrari D, Aragao FA, Albuquerque CE, et al. Reliability and differentiation capability of dynamic and static kinematic measurements of rearfoot eversion in patellofemoral pain. Clin Biomech (Bristol, Avon). 2015;30:144-8.

47. Root ML, Orien WP, Weed JH. Normal and abnormal function of the foot: Clin Biomech. Bristol: Avon; 1977.

48. Farrokhi S, O'Connell M, Fitzgerald GK. Altered gait biomechanics and increased knee-specific impairments in patients with coexisting tibiofemoral and patellofemoral osteoarthritis. Gait Posture. 2015;41:81-5.

49. Teng HL, MacLeod TD, Link TM, Majumdar S, Souza RB. Higher knee flexion moment during the second half of the stance phase of gait is associated with magnetic resonance imaging progression of patellofemoral joint osteoarthritis. J Orthop Sports Phys Ther. 2015:1-32.

\section{Ready to submit your research? Choose BMC and benefit from:}

- fast, convenient online submission

- thorough peer review by experienced researchers in your field

- rapid publication on acceptance

- support for research data, including large and complex data types

- gold Open Access which fosters wider collaboration and increased citations

- maximum visibility for your research: over $100 \mathrm{M}$ website views per year

At BMC, research is always in progress.

Learn more biomedcentral.com/submissions 